Arq. Bras. Med. Vet. Zootec., v.66, n.4, p.969-976, 2014

\title{
Características do sêmen de carneiros Dorper, Santa Inês e sem padrão racial definido, pré e pós-congelação, nos períodos chuvoso e seco
}

[Characteristics of the semen of Dorper, Santa Ines and undefined breed sheep, pre-and post-freezing, in the rainy and dry period]

J.M. Frazão Sobrinho, M.A. Castelo Branco*, A. Sousa Júnior, I.M.R. Nascimento, L.H.C.M. Mota, Y.N.T. Carvalho, S.B. Ferreira, D.N.M. Costa, F.J. Moraes Júnior, J.A.T. Souza

Universidade Federal do Piauí - UFPI - Teresina-PI

\section{RESUMO}

Avaliaram-se as características espermáticas de carneiros Dorper, Santa Inês e sem padrão racial definido, nos períodos chuvoso e seco. Após ser colhido por vagina artificial, o sêmen foi avaliado quanto ao volume, ao turbilhonamento, à motilidade, ao vigor, à morfologia e à concentração, congelado e armazenado em botijão criogênico. Depois de descongelado, foram avaliadas a cinemática espermática, a integridade da membrana plasmática, a integridade do acrossoma e a atividade mitocondrial. Vigor espermático, motilidade total, motilidade progressiva, velocidade em linha reta e defeitos maiores não diferiram entre os períodos chuvoso e seco, porém volume, turbilhonamento, linearidade, retilinearidade e frequência de batimentos de cauda foram mais baixos $(\mathrm{P}<0,05)$ no período seco; já concentração espermática e defeitos totais apresentaram valores mais baixos no período chuvoso. Valores de integridade do acrossoma e da membrana plasmática, bem como o potencial de membrana mitocondrial, foram mais baixos $(\mathrm{P}<0,05)$ no período seco. Conclui-se que os períodos chuvoso e seco influenciam na qualidade espermática de ovinos criados na região Meio-Norte do Brasil e que esses animais têm uma qualidade espermática superior no período chuvoso, quando, portanto, deve ocorrer a criopreservação. Também se observou que, em relação à qualidade espermática, o melhor grupo de carneiros foi o Santa Inês.

Palavras-chave: ovino, características do sêmen, períodos seco e chuvoso, congelação

\begin{abstract}
The sperm characteristics of Dorper, Santa Ines and undefined breed ram in the rainy and dry seasons was assessed. After collection with an artificial vagina, the semen was evaluated for volume, turbulence, motility, viability, morphology and concentration, frozen and stored in a cryogenic cylinder. After thawing kinematic sperm, plasma membrane integrity, acrosome integrity and mitochondrial activity were evaluated. Sperm vigor, total motility, progressive motility, straight line speed and larger line defects did not differ between the rainy and dry seasons, however, volume, turbulence, linearity, straightness and frequency of tail beats were lower $(P<0.05)$ in the dry period, and sperm concentration and total defects showed lower values during the rainy season. Values for acrosome integrity and plasma membrane and mitochondrial membrane potential were lower $(P<0.05)$ in the dry season. It is concluded that the wet and dry period influence sperm quality in ram raised in the Mid - North region of Brazil, with a higher sperm quality in the rainy season, thus suggesting cryopreservation for that period. Also, the better ram group regarding sperm quality, was the Santa Ines.
\end{abstract}

Keyword: sheep, seminal characteristics, freezing

Recebido em 5 de fevereiro de 2013

Aceito em 13 de fevereiro de 2014

*Autor para correspondência (corresponding author)

E-mail: marlon704@gmail.com 


\section{INTRODUÇÃO}

O sêmen é composto por duas frações distintas: o plasma seminal e os espermatozoides, que nos ovinos correspondem a cerca de $30 \%$ do volume total, e pode ser utilizado nas biotécnicas da reprodução in natura, resfriado ou congelado (Gonçalves et al., 2008). A qualidade do sêmen está relacionada à avaliação das características seminais quantitativas e qualitativas (Ibrahim, 1997). Parâmetros do sêmen de carneiros podem ser influenciados por vários fatores, como raça, variabilidade individual, mês e período do ano, fotoperíodo, condições alimentares e temperatura (Pacheco et al., 2009).

Apesar da aplicação comercial generalizada da inseminação artificial com sêmen congelado de ovinos (Salamon e Maxwell, 2000), a fertilidade após a inseminação cervical de ovelhas com sêmen congelado permanece variável e problemática. As diferenças na fertilidade entre carneiros e entre ejaculados de um mesmo carneiro podem ser atribuídas às modificações dos componentes do plasma seminal, aos períodos de congelamento do sêmen (seco ou chuvoso) e à disponibilidade de alimentos que comprometem a viabilidade e a fertilidade (O'Meara et al., 2005). Além disso, parição resultante de sêmen ovino congelado semelhante àquelas com sêmen fresco raramente tem sido obtida após a inseminação artificial cervical (Salamon e Maxwell, 2000).

Houve algumas melhorias nos componentes diluentes para sêmen ovino congelado desde a incorporação rotineira de gema de ovo ou de leite com glicerol como agentes para proteger os espermatozoides durante o resfriamento e o congelamento, respectivamente. Os dois diluentes mais utilizados para a diluição précongelação do sêmen de carneiros são baseados em Tris, frutose, gema de ovo e glicerol (Salamon e Visser, 1973).

Estudos feitos em carneiros Santa Inês (Quirino, 2006) demonstraram variação na qualidade seminal ao longo do ano, pois observaram-se diminuição da motilidade e do vigor e aumento no percentual de patologias nos meses de temperatura mais elevada. As variações na qualidade seminal do sêmen congelado de carneiros podem ser avaliadas por diferentes técnicas, entre elas sondas fluorescentes, teste de termorresistência e cinética espermática. A utilização de sondas fluorescentes tem por objetivo pesquisar incidência de lesões das membranas plasmática e acrossomal, bem como de atividade mitocondrial, durante o processo de congelação/descongelação do sêmen (D’Alessandro e Martemucci, 2003).

Este trabalho teve como objetivo avaliar as características do sêmen e a integridade de espermatozoides de ovinos Dorper, Santa Inês e sem padrão racial definido (SPRD), colhidos nos períodos chuvoso e seco no estado do Piauí.

\section{MATERIAL E MÉTODOS}

O experimento, aprovado pelo Comitê de Ética e Experimentação Animal da Universidade Federal do Piauí, sob o n.003/2011, foi realizado no Laboratório de Biotecnologia da Reprodução Animal da referida instituição, localizado no município de Teresina, município situado a $5^{\circ} 5^{\prime} 21^{\prime \prime} \mathrm{S}$ de latitude, longitude $42^{\circ} 48^{\prime} 7^{\prime \prime} \mathrm{W}$, com altitude de 72 metros. O clima na região é do tipo quente e úmido, com dois períodos bem definidos, chuvoso e seco (Medeiros, 1996). O experimento foi realizado, no período chuvoso, entre os meses de janeiro/2012 e abril/2012, e, no período seco, entre os meses de agosto/2012 e novembro/2012. No período chuvoso, a região apresentou precipitação pluviométrica média de $400 \mathrm{~mm}$, temperatura média de $31^{\circ} \mathrm{C}$ e umidade relativa do ar de $60 \%$. Já no período seco, a precipitação pluviométrica média foi de $20 \mathrm{~mm}$, a temperatura média de $37^{\circ} \mathrm{C}$ e a umidade relativa do ar de $35 \%$.

Foram utilizados 18 carneiros adultos, sendo seis Dorper (DP), seis Santa Inês (SI) e seis sem padrão racial definido (SPRD), com idades de 34 anos, pesando entre 50-60kg e com escore corporal 3 - 4 (escala $1-5$ ). Todos os carneiros foram avaliados quanto à saúde geral e à integridade dos órgãos reprodutivos. Durante o experimento, os carneiros foram mantidos sob regime extensivo, com acesso a piquetes de capim- mimoso (Axonopus purpusii) e capimmilhã (Digitaria sanguinalis), água e sal mineral à vontade.

Os animais foram submetidos a uma colheita de sêmen por semana, por meio do método de vagina artificial. Imediatamente após a colheita, o sêmen foi avaliado quanto ao volume, ao 
turbilhonamento, ao vigor e à motilidade. Colocaram-se $20 \mu \mathrm{L}$ de sêmen em $2 \mathrm{~mL}$ de formol salino (diluição de 1:200) para posterior avaliação da concentração e morfologia espermática. A concentração espermática $\left(10^{9} \mathrm{sptz} / \mathrm{mL}\right)$ foi realizada por meio da técnica câmara de Neubauer, e a morfologia espermática pela técnica câmara úmida, segundo o CBRA (Manual..., 1998).

As amostras de sêmen foram diluídas em Tris-gema $(3,605 \mathrm{~g}$ de Tris, 2,024g de ácido cítrico, $1,488 \mathrm{~g}$ de frutose, $100 \mathrm{~mL}$ de água destilada, $20 \%$ de gema de ovo e $5 \%$ de glicerol), para uma concentração final de $240 \times 10^{6}$ espermatozoides $/ \mathrm{mL}$. Posteriormente, foram armazenadas em palhetas $(0,25 \mathrm{~mL})$ e congeladas em máquina TK $3000^{\circledR}$ (TK Tecnologia em congelação Ltda., Uberaba, Brasil), na curva de congelação rápida $\left(-0,25^{\circ} \mathrm{C} / \mathrm{min}\right.$, de $25^{\circ} \mathrm{C}$ a $5^{\circ} \mathrm{C}$ e $-20^{\circ} \mathrm{C} / \mathrm{min}$, de $5^{\circ} \mathrm{C}$ a $\left.-120^{\circ} \mathrm{C}\right)$, e, após atingirem $120^{\circ} \mathrm{C}$, as palhetas foram armazenadas em nitrogênio líquido $\left(-196^{\circ} \mathrm{C}\right)$. Depois de 30 dias de armazenamento, as amostras de sêmen foram descongeladas a $37^{\circ} \mathrm{C}$ por 30 segundos e avaliadas quanto à integridade do acrossoma, à integridade da membrana plasmática, à função mitocondrial e à cinemática.

Para avaliação da integridade do acrossoma, utilizou-se o corante isotiocianato de fluoresceína conjugado a Peanut agglutinin (FITC-PNA; Sigma-Aldrich®, St Louis, MO, USA), de acordo com a técnica descrita por Roth et al. (1998), em que uma alíquota de $20 \mu \mathrm{L}$ da solução estoque de FITC-PNA $(1 \mathrm{mg} / \mathrm{mL})$ foi descongelada e adicionada a $480 \mu \mathrm{L}$ de $\mathrm{PBS}$ para obter a concentração final de $100 \mu \mathrm{g} / \mathrm{mL}$. Alíquotas $(20 \mu \mathrm{L})$ desta solução foram colocadas sobre esfregaços de lâminas, as quais foram incubadas por 20 minutos em câmara úmida a $4^{\circ} \mathrm{C}$, na ausência de luz. Após incubação, as lâminas foram enxaguadas duas vezes em PBS refrigerado $\left(4^{\circ} \mathrm{C}\right)$ e colocadas para secagem na ausência de luz. Imediatamente antes da avaliação, $5 \mu \mathrm{L}$ de meio de montagem UCD $(4,5 \mathrm{~mL}$ de glicerol, $0,5 \mathrm{~mL}$ de PBS, $5 \mathrm{mg}$ de azida sódica e $5 \mathrm{mg}$ de p-fenilenodiamina) foram colocados sobre a lâmina e cobertos com lamínula. Foram avaliados 200 espermatozoides por lâmina, com aumento de $1000 x$, sob óleo de imersão, em microscópio de epifluorescência (Olympus, Japão), usando-se filtro de emissão LP $515 \mathrm{~nm}$ e BP 450-490nm para excitação. Os gametas foram classificados como portadores de acrossomas intactos, quando apresentavam a região acrossomal corada com fluorescência verde, ou como acrossoma reagido, quando apresentavam uma faixa verde fluorescente na região equatorial da cabeça espermática ou não apresentavam fluorescência verde em toda região da cabeça.

A função mitocondrial foi determinada pela utilização de um fluorocromo catiônico lipofílico JC-1 (Guthrie e Welch, 2006). Para tanto, alíquotas de $50 \mu \mathrm{L}$ de sêmen pós-descongelado foram diluídas em $150 \mu \mathrm{L}$ de Tris contendo $5 \mu \mathrm{L}$ de JC-1 $(0,15 \mathrm{mM}$ em DMSO $)$ e incubadas por 10 minutos a $38^{\circ} \mathrm{C}$. Um total de 200 espermatozoides foi avaliado em microscópio de epifluorescência (Olympus, Japão), com aumento de 1000x, sob óleo de imersão, usando-se filtro de emissão LP 515nm e BP 450-490nm para excitação. As células coradas em laranja foram classificadas com alto potencial de membrana mitocondrial, e aquelas coradas em verde foram classificadas com baixo potencial de membrana.

Para avaliação da integridade da membrana plasmática, utilizou-se o método de coloração dupla com diacetato de carboxifluoresceína (DCF; Sigma-Aldrich®, St. Louis, MO, USA) e iodeto de propídio (IP; Sigma-Aldrich®, St. Louis, MO, USA), modificado por Coleto et al. (2002), em que alíquotas de $50 \mu \mathrm{L}$ de sêmen pósdescongeladas foram diluídas em $150 \mu \mathrm{L}$ de Tris contendo $5 \mu \mathrm{L}$ de DCF $(0,46 \mathrm{mg} / \mathrm{mL}$ em DMSO $)$ e $20 \mu \mathrm{L}$ de IP $(0,5 \mathrm{mg} / \mathrm{mL}$ em PBS $)$ e incubadas por 10 minutos a $38^{\circ} \mathrm{C}$. Um total de 200 espermatozoides foi avaliado em microscópio de epifluorescência (Olympus, Japão), com aumento de 400x, usando-se filtro de emissão DBP 580630nm e excitação DBP 485/20nm. Eles foram classificados com membrana intacta, quando se apresentavam corados em verde, e com membrana danificada, quando corados em vermelho.

A cinemática espermática foi avaliada por meio de um sistema analisador de espermatozoide auxiliado por computador (CASA). O CASA consistiu de um sistema de microscopia óptica de contraste de fase (Nikon TM H5505, Eclipse 50i, Japão), com iluminação estroboscópica, e uma fase quente a $37^{\circ} \mathrm{C}$, uma câmera de vídeo (Basler Visão Tecnologie TM A312FC, Ahrensburg, Alemanha) e um PC com o analisador de 
esperma Classe TM software (Microptics, SL, versão 3.2.0, Barcelona, Espanha). As variáveis cinéticas dos espermatozoides foram avaliadas após a diluição da amostra em citrato de sódio $2,94 \%$ (v/v) e incubação num banho de água a $37^{\circ} \mathrm{C}$ durante $5 \mathrm{~min}$. As variáveis avaliadas foram: velocidade curvilinear (VCL $-\mu \mathrm{m} / \mathrm{s}$ ), velocidade em linha reta (VSL $-\mu \mathrm{m} / \mathrm{s}$ ), velocidade média do percurso (VAP- $\mu \mathrm{m} / \mathrm{s}$ ), linearidade (LIN - \%), retilinearidade (STR-\%), deslocamento lateral de cabeça (ALH - $\mu \mathrm{m}$ ) e frequência de batimento cruzado (BCF-Hz), para cada espermatozoide analisado.

O delineamento experimental utilizado foi parcela subdividida, considerando-se os efeitos dos grupos raciais, a época do ano e as interações entre eles. Após o teste de Bartlett para constatar a homogeneidade entre as variâncias, algumas variáveis (volume, vigor, VCL, VSL, VAP, LIN, mitocôndria e acrossoma), por não apresentarem normalidade ou homogeneidade, sofreram transformações dos dados. Os dados tabulados foram analisados por meio do software Statistical Analysis System (SAS, 2002) e do teste de comparação de médias de Tukey, com significância de $5 \%(\mathrm{P}<0,05)$.

\section{RESULTADOS E DISCUSSÃO}

Entre as variáveis estudadas, referentes aos aspectos físicos e morfológicos do sêmen, apenas o vigor apresentou interação entre o período do ano e os grupos de carneiros estudados. Evidenciou-se que carneiros Santa Inês e os sem padrão racial definido não apresentaram diferença significativa $(\mathrm{P}>0,05)$ em relação ao vigor, nos períodos chuvoso e seco. Entretanto, os carneiros Dorper apresentaram um vigor inferior no período seco. Isso pode ter sido pelo fato de que os carneiros Dorper apresentam genótipo exótico, por isso têm um menor grau de adaptabilidade. E essa baixa adaptabilidade pode expressar-se em aumento do estresse térmico (Cezar et al., 2004). Segundo Coelho et al. (2008), temperaturas elevadas podem interferir negativamente na temperatura testicular e, consequentemente, na qualidade espermática de pequenos ruminantes.

Os resultados em relação às demais características do sêmen referentes às variáveis físicas e morfológicas, nas quais não houve interação entre os grupos de animais e os períodos, encontram-se na Tab. 1. Observou-se diferença estatística para volume, turbilhonamento, motilidade, concentração espermática, defeitos maiores e defeitos totais entre os períodos chuvoso e seco independentemente do grupo de carneiros. Os valores no período chuvoso foram superiores àqueles no período seco, exceto para concentração espermática, na qual os valores foram superiores no período seco, provavelmente por acréscimo do volume seminal no período chuvoso em razão do aumento nas secreções epididimárias e glandulares; no período seco, a redução dessas secreções resulta em uma concentração maior de célula por volume de ejaculado (Leal et al., 1998).

Tabela 1. Variáveis físicas (volume, turbilhonamento, motilidade), concentração espermática e patologias do sêmen de ovinos nos períodos chuvoso e seco

\begin{tabular}{l|cc|ccc}
\hline \multirow{2}{*}{ Variáveis } & \multicolumn{2}{c|}{ Período } & \multicolumn{3}{c}{ Carneiros } \\
\cline { 2 - 6 } & Chuvoso & Seco & SPRD & SI & DP \\
\hline Volume (mL) & $0,82 \mathrm{a} \pm 0,1$ & $0,71 \mathrm{~b} \pm 0,1$ & $0,80 \mathrm{a} \pm 0,2$ & $0,87 \mathrm{a} \pm 0,1$ & $0,63 \mathrm{~b} \pm 0,3$ \\
Turbilhonamento (1-5) & $3,34 \mathrm{a} \pm 0,5$ & $3,10 \mathrm{~b} \pm 0,3$ & $3,29 \mathrm{a} \pm 0,1$ & $3,26 \mathrm{a} \pm 0,6$ & $3,12 \mathrm{a} \pm 0,4$ \\
Motilidade (\%) & $77,41 \mathrm{a} \pm 3,6$ & $74,28 \mathrm{~b} \pm 4,5$ & $76,87 \mathrm{a} \pm 3,0$ & $77,33 \mathrm{a} \pm 4,6$ & $73,33 \mathrm{a} \pm 3,5$ \\
Concentração (10 & $2,11 \mathrm{a} \pm 0,4$ & $2,52 \mathrm{~b} \pm 0,3$ & $2,3 \mathrm{a} \pm 0,5$ & $2,4 \mathrm{a} \pm 0,1$ & $2,14 \mathrm{a} \pm 0,8$ \\
sptz/mL) & & & & \\
Defeitos maiores (\%) & $4,54 \mathrm{a} \pm 0,9$ & $4,00 \mathrm{a} \pm 0,6$ & $3,67 \mathrm{a} \pm 0,3$ & $3,90 \mathrm{a} \pm 0,5$ & $5,23 \mathrm{a} \pm 0,7$ \\
Defeitos totais (\%) & $21,40 \mathrm{a} \pm 3,5$ & $29,61 \mathrm{~b} \pm 2,6$ & $25,80 \mathrm{a} \pm 4,5$ & $24,30 \mathrm{a} \pm 2,9$ & $26,43 \mathrm{a} \pm 3,7$ \\
\hline
\end{tabular}

Médias seguidas de letras diferentes na mesma linha diferem significativamente $(\mathrm{P}<0,05)$ pelo teste Tukey. SPRD - sem padrão racial definido; SI - Santa Inês; DP - Dorper. 
Ao se compararem os grupos de carneiros, independentemente do período, observa-se que não houve diferença estatística para as variáveis estudadas, exceto para volume, no qual o grupo de animais Dorper apresentou volume inferior aos demais grupos estudados. Esses dados corroboram os observados por Rodrigues (2004).

As variáveis do sêmen dos grupos de animais Dorper e Santa Inês, especialmente a motilidade espermática e a porcentagem de espermatozoides anormais, diferiram de estudos realizados também na região Nordeste (Silva e Nunes, 1984; Souza et al., 2007). Essa redução na motilidade pode ser decorrente do maior percentual de espermatozoides anormais (Bearden et al., 2004). Altas temperaturas afetam, especialmente, a motilidade, que sofre uma queda acentuada, e o aumento da porcentagem de espermatozoides anormais (Coelho et al., 2008).Entre as variáveis da cinemática espermática, apenas a velocidade curvilinear (VCL) e a velocidade média de percurso (VAP) apresentaram interação entre o período do ano e os grupos estudados. Os carneiros Dorper e SPRD apresentaram maior VCL e VAP no período seco, e o Santa Inês apresentou maior VCL e VAP no período chuvoso. Quando comparados os grupos de carneiros entre si, em um mesmo período, observa-se que, no período seco, o Dorper destacou-se dos demais com relação à VCL e à VAP. Já no período chuvoso, o Santa Inês apresentou VCL e VAP superiores às dos demais grupos de carneiros.

Os dados referentes à análise computadorizada do sêmen descongelado e avaliado por meio do sistema CASA, no qual não houve interação entre períodos e os grupos de carneiros, encontram-se dispostos na Tab. 2. Os resultados de motilidade total (MOT) e motilidade progressiva (MP) apresentaram o mesmo padrão, não havendo diferença entre os períodos chuvoso e seco. Esses dados corroboram estudos anteriores que afirmam haver pouca e/ou nenhuma influência do período climático sobre a condição reprodutiva de ovinos (Simplício, 2008). Ao se compararem os grupos de carneiros, independentemente dos períodos, observa-se que os carneiros Santa Inês e Dorper apresentam valores de MOT e MP superiores aos animais SPRD. A MOT e a MP encontradas neste trabalho foram superiores às descritas por Rodello (2006), porém os valores de VCL, VSL e VAP estão inferiores aos observados por Joshi et al. (2005). Este resultado pode ser em virtude de vários fatores, tais como: diluidor utilizado, protocolo de congelação, ou mesmo fatores ligados aos animais (El-Alamy e Foote, 2001).

Tabela 2. Cinética espermática em sêmen descongelado de ovinos nos períodos chuvoso e seco

\begin{tabular}{c|cc|ccc}
\hline \multirow{2}{*}{ Variáveis } & \multicolumn{2}{|c}{ Período } & \multicolumn{3}{c}{ Carneiros } \\
\cline { 2 - 6 } & Chuvoso & Seco & SPRD & SI & DP \\
\hline MOT $(\%)$ & $45,69 \mathrm{a} \pm 8,3$ & $47,11 \mathrm{a} \pm 10,3^{\mathrm{a}}$ & $42,79 \mathrm{a} \pm 7,3$ & $47,39 \mathrm{~b} \pm 5,9$ & $49,02 \mathrm{~b} \pm 12,3$ \\
MP $(\%)$ & $29,1 \mathrm{a} \pm 5,6$ & $32,3 \mathrm{a} \pm 4,8$ & $27,2 \mathrm{a} \pm 5,3$ & $35,7 \mathrm{~b} \pm 4,2$ & $37,3 \mathrm{~b} \pm 7,4$ \\
VSL $(\mu \mathrm{m} / \mathrm{s})$ & $30,68 \mathrm{a} \pm 9,4$ & $28,47 \mathrm{a} \pm 5,7$ & $24,00 \mathrm{a} \pm 4,6$ & $33,17 \mathrm{bc} \pm 7,5$ & $31,54 \mathrm{c} \pm 8,6$ \\
LIN $(\%)$ & $58,66 \mathrm{a} \pm 7,6$ & $51,05 \mathrm{~b} \pm 8,5$ & $53,31 \mathrm{a} \pm 9,5$ & $59,47 \mathrm{~b} \pm 11,0$ & $51,78 \mathrm{a} \pm 12,5$ \\
STR $(\%)$ & $75,84 \mathrm{a} \pm 8,6$ & $69,83 \mathrm{~b} \pm 10,6$ & $71,93 \mathrm{a} \pm 9,7$ & $76,45 \mathrm{~b} \pm 8,6$ & $70,13 \mathrm{a} \pm 7,4$ \\
BCF $(\mathrm{Hz})$ & $7,96 \mathrm{a} \pm 2,4$ & $7,49 \mathrm{~b} \pm 1,5$ & $7,33 \mathrm{a} \pm 3,4$ & $8,11 \mathrm{bc} \pm 2,7$ & $7,72 \mathrm{ac} \pm 2,5$ \\
\hline
\end{tabular}

Médias seguidas de letras diferentes na mesma linha diferem significativamente $(\mathrm{P}<0,05)$ pelo teste de Tukey. SPRD - sem padrão racial definido; SI - Santa Inês; DP - Dorper; MOT- motilidade total; MP - motilidade progressiva; VSL - velocidade em linha reta; LIN - linearidade; STR - retilinearidade; BCF - frequência de batimentos de cauda.

$\mathrm{O}$ alto percentual de espermatozoides mortos durante o congelamento, comprovado pela MT (42,9 a 60\%), pode ter contribuído para os referidos valores de VCL, VSL e VAP, uma vez que o espermatozoide morto libera enzimas (oxidases), nocivas às células vivas (Joshi et al., 2005). Segundo Watson (2000), durante o congelamento, pode ocorrer a ruptura entre os elementos do axonema da cauda do espermatozoide, o que pode tanto comprometer a motilidade como interferir na qualidade do movimento. Os valores de LIN, STR e BCF são superiores no período chuvoso, porém, ao se compararem os grupos, independentemente do período, observa-se que o Santa Inês apresenta valores superiores as demais para as mesmas características de LIN, STR e BCF.

Entre as variáveis da estrutura espermática, apenas a integridade do acrossoma teve interação 
entre os períodos e os grupos de carneiros estudados. Os resultados das demais variáveis, nas quais não houve interação, encontram-se na Tab. 3. A integridade do acrossoma foi significativamente maior $(\mathrm{P}<0,05)$ no período chuvoso para os três grupos de carneiros estudados. Ao se compararem os resultados nos períodos, observou-se que o Dorper apresentou melhor integridade de acrossoma $(51,66 \pm 10,5)$ no período chuvoso; já no período seco, quem obteve melhor integridade de acrossoma foi o Santa Inês $(30,62 \pm 8,4)$. Sousa et al. (2010) encontraram valores superiores a $90 \%$ de acrossoma íntegro no sêmen resfriado de ovinos Dorper. A integridade da membrana plasmática e a atividade mitocondrial foram significativamente maiores $(\mathrm{P}<0,05)$ no período chuvoso. E os animais Santa Inês, independentemene do período, apresentaram maior integridade de membrana plasmática e atividade mitocondrial.

Os índices de fertilidade são mais baixos, com as características observadas no período de seca, uma vez que a presença de membranas íntegras é um pré-requisito para os eventos relacionados ao processo de fertilização, como a capacitação espermática, a penetração no revestimento do oócito, a ligação à zona pelúcida e a fusão com o oolema (Soares e Guerra, 2009). A avaliação da estrutura espermática é importante para predizer a fertilidade de um reprodutor, pois a observação da motilidade é subjetiva e limitada e não corresponde unicamente à habilidade de fertilização dos gametas masculinos, uma vez que espermatozoides podem apresentar movimento, mas podem não estar íntegros quanto às membranas (Maziero et al., 2009).

A redução da temperatura durante o processo de congelação do sêmen afeta a bomba de $\mathrm{Na}+/ \mathrm{K}+$, provocando despolarizações parciais das membranas, o que as torna permeáveis ao cálcio. Isto induz a uma vesiculação prematura da membrana acrossomal (Bicudo et al., 2007). Esta afirmação foi confirmada por Azevedo (2006) quando, ao avaliar o sêmen ovino em todas as fases do processamento, concluiu que a congelação/descongelação causa mais danos do que a refrigeração e constatou ainda haver diferenças entre indivíduos relacionadas à criorresistência e à criocapacitação espermática.

Tabela 3. Características estruturais da célula espermática do sêmen congelado de ovinos nos períodos chuvoso e seco

\begin{tabular}{c|cc|ccc}
\hline \multirow{2}{*}{ Variáveis } & \multicolumn{2}{|c|}{ Período } & \multicolumn{3}{c}{ Carneiros } \\
\cline { 2 - 6 } & Chuvoso & Seco & SPRD & SI & DP \\
\hline $\begin{array}{c}\text { Integridade da membrana } \\
\text { plasmática (\%) }\end{array}$ & $46,87 \mathrm{a} \pm 11,7$ & $36,87 \mathrm{~b} \pm 9,7$ & $39,14 \mathrm{a} \pm 8,4$ & $45,29 \mathrm{~b} \pm 8,7$ & $41,18 \mathrm{a} \pm 5,7$ \\
Atividade mitocondrial (\%) & $48,41 \mathrm{a} \pm 8,6$ & $39,16 \mathrm{~b} \pm 7,5$ & $34,12 \mathrm{a} \pm 6,9$ & $41,60 \mathrm{bc} \pm 8,6$ & $40,64 \mathrm{c} \pm 8,6$ \\
\hline
\end{tabular}

Médias seguidas de letras diferentes na mesma linha diferem significativamente $(\mathrm{p}<0,05)$ pelo teste de Tukey. SPRD sem padrão racial definido; SI - Santa Inês; DP - Dorper.

\section{CONCLUSÕES}

Conclui-se que ovinos Santa Inês, Dorper e sem padrão racial definido, criados no estado do Piauí em condições de alimentação adequadas, sofrem ação da condição climática, sendo o período seco prejudicial a várias características do sêmen de espermatozoides submetidas ao processo de criopreservação. No período chuvoso, os carneiros apresentam melhores características do sêmen, o que sugere criopreservação seminal nesse período. E, ainda, os carneiros Santa Inês apresentaram uma melhor qualidade espermática.

\section{REFERÊNCIAS}

AZEVEDO, H.C. Integridade e funcionalidade dos espermatozoides ovinos submetidos à criopreservação após a incorporação de colesterol, desmosterol, ácido oleico-linoleico e alfalactoalgumina. 2006. 218f. Tese (Doutorado) - Faculdade de Medicina Veterinária e Zootecnia, Universidade Estadual Paulista, Botucatu.

BEARDEN, J.H.; FUQUAY, J.W.; WILLARD, S.T. Applied animal reproduction. 6.ed. Upper Saddle River: Pearson-Prentice Hall, 2004. 427p.

BICUDO, S.D.; AZEVEDO, H.C.; MAIA S.M. et al. Avanços na criopreservação do sêmen ovino visando sua aplicação em programas de inseminação artificial e em biotecnologias com embriões. Acta Sci. Vet., v.35, p.787-798, 2007. 
CEZAR, M.F.; SOUZA, B.B.; SOUZA, W.H. et al. Avaliação de parâmetros fisiológicos de ovinos Dorper, Santa Inês e seus mestiços perante condições climáticas do trópico semi-árido nordestino. Ciênc. agrotec., v.28, p.614-620, 2004.

COELHO, L.A.; SASA, A.; BICUDO, S.D.; BALIEIRO, J.C.C. Concentrações plasmáticas de testosterona, triiodotironina (T3) e tiroxina (T4) em bodes submetidos ao estresse calórico. Arq. Bras. Med. Vet. Zootec., v.60, p.1338-1345, 2008.

COLETO, Z.F.; GUERRA, M.M.P.; BATISTA, A.M. Avaliação do sêmen congelado de caprinos com drogas fluorescentes. Rev. Bras. Med. Vet., v.24, p.101-104, 2002.

D'ALESSANDRO, A.G.; MARTEMUCCI, G Evaluation of seasonal variations of semen freezability in Leccese ram. Anim. Reprod. Sci., v.79, p.93-102, 2003.

EL-ALAMY, M.A.; FOOTE, R.H. Freezability of spermatozoa from Finn and Dorset rams in multiple semen extenders. Anim. Reprod. Sci., v.65, p.245-254, 2001.

GONÇALVES, P.B.D.; FIGUEIREDO, J.R.; FREITAS, V.J.F. Biotécnicas aplicadas à reprodução animal. 2. Ed. Cap. 5. Sao Paulo: Roca, 2008. p.83103.

GUTHRIE, H.D.; WELCH G.R. Determination of intracellular reactive oxygen species and high mitochondrial membrane potential in Percoll-treated viable boar sperm using fluorescence-activated flow cytometry. J. Anim. Sci., v.84, p.2089-2100, 2006.

IBRAHIM, S. Seasonal variations in semen quality of local and crossbred rams raised in United Arab Emirates. Anim. Reprod. Sci., v.49, p.161-167, 1997.

JOSHI, A.; BAG, S.; NAQVI, S. et al. Effect of postthawing incubation on sperm motility and acrosomal integrity of cryopreserved Garole ram semen. Small Rum. Rese., v.56, p.231-238, 2005.

LEAL, T.M.; REIS, J.C.; GIRÃO, R.N. et al. Características do sêmen de carneiros deslanados da raça Santa Inês criados no nordeste brasileiro. Cienc. Vet. Trop., v.1, p.49-54, 1998.

MANUAL para exame andrológico e avaliação do sêmen animal. Belo Horizonte: Colégio Brasileiro de Reprodução Animal. 2.ed., 1998. 49p.

MAZIERO, R.R.D.; CRESPILHO, A.M.; FREITASDELL'AQUA, C.P. et al. Análise de sêmen bovino e sua relação com a fertilidade. Rev. Bras. Reprod. Anim., v.6, p.5-10, 2009.
MEDEIROS, J.C.; AMORIM NETO, M.; BELTRÃO, N.E.M. et al. Zoneamento para a cultura do algodão no Nordeste. I. Algodão arbóreo. Campina Grande: Embrapa-CNPA, 1996. 31p (EMBRAPA-CNPA. Boletim de Pesquisa, 31).

O'MEARA C.M.; HANRAHAN J.P.; DONAVAN A. et al. Relationship between in vitro fertilisation of ewe oocytes and the fertility of ewes following cervical artificial insemination with frozen thawed ram semen. Theriogenology, v.64, p.1797-1808. 2005.

PACHECO, A.; OLIVEIRA, M.A.F.; QUIRINO, C.R. et al. Características seminais de carneiros da raça Santa Inês na pré-puberdade, puberdade e póspuberdade. Ars Veterinária, v.25, p.090-099, 2009.

QUIRINO, C.R.; PACHECO, A.; AFONSO, V.A.C. et al. Efeito da estação do ano sobre as características físicas do sêmen, biometria testicular e corporal de ovinos da raça Santa Inês na região norte fluminense. In: ZOOTEC 2006, Recife, PE. Anais... Recife, PE: ABZ, 2006, CD-ROM.

RODELLO, L. Validação de Sistema Automatizado de Refrigeração e Congelação de Sêmen Ovino. Tese (Mestrado em Medicina Veterinária), Faculdade de Medicina Veterinária e Zootecnia, Universidade Estadual Paulista-UNESP, Botucatu.

RODRIGUES A.L.R. Avaliação do Testículo e da cauda do epidídimo de carneiros na pré-puberdade, na maturidade sexual, no criptorquidismo e na insulação escrotal: concentração de testosterona plasmática e tecidual, histamina, fator de necrose tumoral e óxido nítrico. Botucatu-SP: Universidade Estadual Paulista, 2004. 106f. Tese (Doutorado) Faculdade de Medicina Veterinária e Zootecnia., Campus de Botucatu, 2004.

ROTH, T.L.; WEISS, R.B.; BUFF, J.L. et al. Heterologous in vitro fertilization and sperm capacitation in an endangered African antelope, the Scimitar-Horned Oryx (Oryx dammah). Biol. Reprod., v.58, p.475-482, 1998.

SALAMON, S.; VISSER, D. Fertility test of frozen boar spermatozoa. Australian J. Biol. Sci., v.26, p.291, 1973.

SALAMON, S. AND MAXWELL, W.M.C. Storage of ram semen. Anim. Reprod. Sci., v.62, p.77-111. 2000.

SILVA, A.E.D.F.; NUNES, J.F. Estacionalidade na atividade sexual e qualidade do sêmen nos ovinos deslanados das raças Santa Inês e Somalis. Revista Brasileira de Reprodução Animal, v.8, n.4, p.207-214, 1984. 
SIMPLÍCIO, A.A. Estratégias de manejo reprodutivo como ferramenta para prolongar o período de oferta de carnes caprina e ovina no Brasil. Tecnol. Cienc. Agrop., v.2, p.29-39, 2008.

SOARES, A.T.; GUERRA, M.M.P. Efeitos da criopreservação sobre a viabilidade espermática. Tecnol. Cienc. Agrop., v.3, p.53-63, 2009.

SOUSA, C.E.A.; ARAÚJO, A.A.; OLIVEIRA, J.T.A. et al. Reproductive development of Santa Inês rams during the first year of life: body and testis growth, testosterone concentrations, sperm parameters, age at puberty and seminal plasma proteins. Reprod. Domestic Anim., v.45, p.644-653, 2010.
SOUZA, J.A.T.; CAMPELO, J.E.G.; MACEDO, N.A. et al. Biometria testicular, características seminais, libido e concentração de testosterona em ovinos da raça Santa Inês, criados a campo, na microrregião de Campo Maior, Piauí. Cienc. Vet. Trop., v.10, p.21-28, 2007.

WATSON, P.F. The causes of reduced fertility with cryopreserved semen. Anim. Reprod. Sci., v.61, p.48192, 2000. 